\title{
Feedback Distortion for Rehabilitation: Gauging Perceived Physical Effort
}

\author{
Matthew Fagan ${ }^{1}$, Yoky Matsuoka ${ }^{2,3, *}$, and Roberta Klatzky ${ }^{4}$ \\ Carnegie Mellon University \\ Pittsburgh, PA 15213
}

\begin{abstract}
We examined subjects' perceptions of physical effort in the presence of feedback distortion as they were asked to resist forces produced by a haptic device. Subjects pressed against a simulated spring with the index finger until they reached a target force, which they held for a designated time duration. Visual feedback of the target force level and the designated time duration was provided. On some trials, the visual feedback of force was distorted by $7 \%$ to $22 \%$ so that subjects produced that much more force to get to the same visual target force. Subjects rated their perceived physical effort for each trial. Our regression analyses showed that rated effort was strongly predicted by the visual feedback of force regardless of the actual force produced. The length of time the force was produced only weakly predicted the rated effort. These results will be used to construct virtual rehabilitation environments.
\end{abstract}

\section{Introduction}

The long-term goal of our program on feedback distortion for rehabilitation is to develop therapeutic methods utilizing a virtual environment for distorting patients' perceptions of their environment, in order to encourage them to increase their range of motion or force production without patients' awareness. Patients undergoing mobility rehabilitation after stroke or brain injuries would be the primary candidates for this type of therapy. Our previous experiments with virtual environments have focused on determining the range of distortion that can be used in a therapeutic setting without patients' awareness. In the same virtual environment as a therapeutic condition, our preliminary results indicated that the Just Noticeable Differences

1. Computer Science Department

2. The Robotics Institute

3. Department of Mechanical Engineering

4. Department of Psychology

* Corresponding author (yoky@cs.cmu.edu)
(JNDs) of healthy young adults for force and position are $14.4 \%$ and $18.0 \%$ respectively [1]. Furthermore, we demonstrated how these JNDs could be extended to $35.9 \%$ when visual representations of force and position are distorted [9].

While sensory limitations such as JNDs are crucial in determining the distortion level to be used during rehabilitation, higher-level cognitive factors such as patients' sense of physical effort or exertion could also influence our rehabilitation guidelines. "Effort," for these purposes, is defined in terms of work output and metabolic expense, which may include cardio-respiratory variables and other metabolic measurements [6]. In our case, we are using perceived effort as a gauge of the amount of work that a person believes he or she has done: the sense of fatigue or exertion after completing a trial.

Previous empirical research on perceived physical effort has focused on occupational and recreational tasks as personnel specialists rated physical effort during each task $[6,7]$. They found a high correlation $(\mathrm{r}=0.81)$ between the metabolic costs of the occupational tasks and the mean effort ratings (based on a 7-graded scale) given by subjects. The study was repeated using subjects who were not personnel specialists, and correlations remained strong (from $r=0.70$ to $r=0.80$ ). Standard deviations in the effort ratings were quite low among the subjects (from 0.06 to 1.60 ), indicating a high degree of inter-rater agreement. Williams and Purewal [10] experimented with subjects similar in age and socioeconomic status to the target subjects of our study and they found that using their own "Effort Sense Rating Scale," in both aerobic and anaerobic situations, subjects were able to rate activities with a $77.5 \%$ correlation to measured fitness variables.

A significant study of the human factors affecting perceived effort is presented in [2], utilizing the 10-point Borg Ratings of Perceived Exertion (RPE) Scale. Borg's RPE rating of "10" represents the point of complete exhaustion. He has shown that psychological factors such as personality, fear, and anxiety affect somatic responses and the perceived exertion levels. $\mathrm{Lu}$ [8] found that factors such as personality and social 
desirability could affect RPE, but contribute a different amount at different exercise intensities. Specifically, psychological variables had the most effect at low metabolic activity levels. Physiologically, Cain [3] found that blood occlusions during hand contractions accelerated the perceived fatigue most in the first 45 seconds of exertion. This shows that fatigue caused by physiological changes could change the perceived effort especially at the beginning of trials. In addition, he found that non-dominant hands fatigued faster than dominant hands.

In our experiment, we studied healthy individuals' perception of physical effort when they received distorted feedback about their force production levels. We hypothesized that subjects would be relatively insensitive to the actual force produced in the presence of distorted visual feedback of force representation. We varied both the force magnitude and the time duration of the force production for to determine the interplay of force and time on perceived physical effort. Control experiments were conducted with no visual feedback of force. Using linear and multiple regression analyses, we assessed the separate contributions of force, time, and distortion to perceived physical effort. Our ultimate goal is to develop a rehabilitative method that will be transferable to disabled populations. We will use the results from healthy individuals as a baseline tool to construct the therapeutic paradigms.

\section{Methods}

\subsection{Visual feedback experiments}

We used a PHANTOM ${ }^{\mathrm{TM}}$ Premium 1.5 (Sensable Technologies, Woburn, MA) with a customized finger coupler as our haptic display. Nine healthy subjects between the ages of 20 and 32 ( 4 males, 5 females) participated in the set experiments with full visual feedback. All were right-handed and none had any known neurological disorders. Subjects sat on a chair facing the computer monitor and the robot, so that they were viewing the visual feedback bar on the monitor from approximately $12 \mathrm{~cm}$ above and $46 \mathrm{~cm}$ in front (see Figure 1). Subjects' right arm was placed on a side support, and their index finger was secured with a finger splint so that the index finger could only be moved at the metacarpo-phalangeal joint, as shown in Figure 2. The finger's range of motion was limited by a hard stop imposed by the robot's force at the 0 degree flexion point and by a physical obstacle at the 90-degree flexion point. Subjects held a post with the remainder of their fingers to secure the hand position, and the subject's

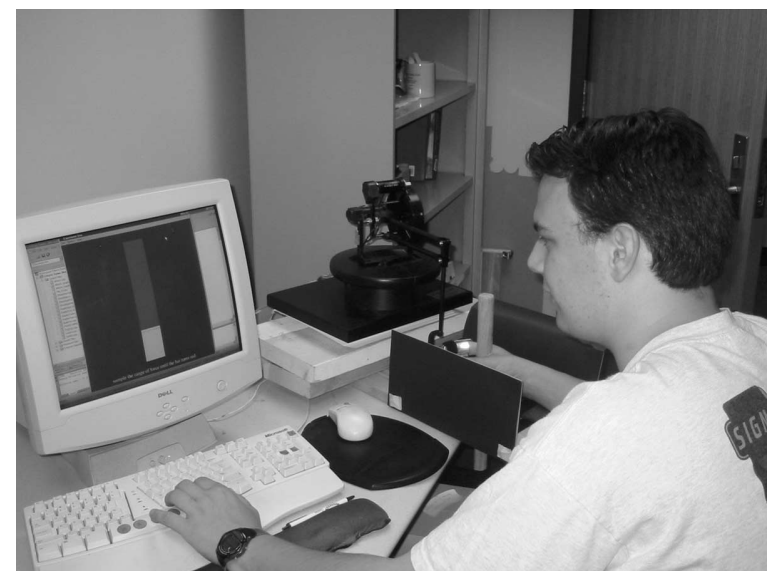

Figure 1. For our experimental setup, subjects sat in front of the computer monitor and the PHANTOM $^{\mathrm{TM}}$. During the experiment, the subject's hand and fingers were obscured from view with an opaque paper hood (not shown).

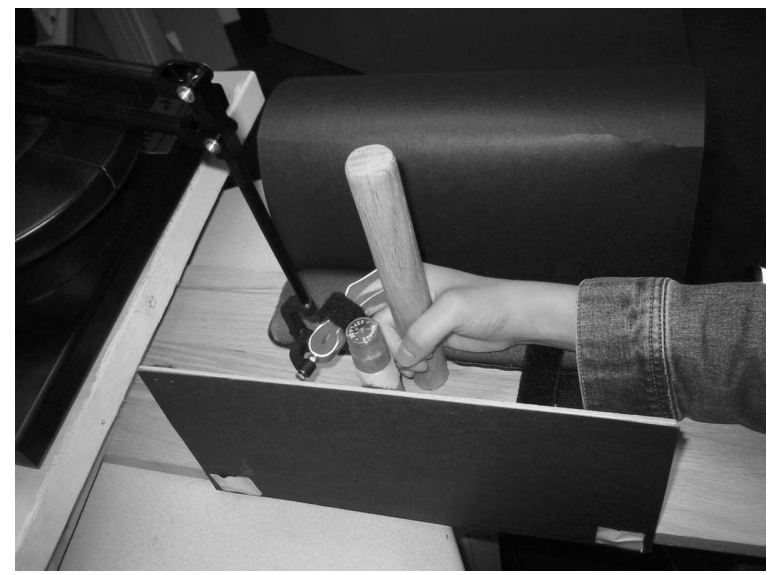

Figure 2. Subjects' index finger was secured with a finger splint and then to the end of the PHANTOM $^{\mathrm{TM}}$ so that they could only move their metacarpo-phalangeal joint. Subjects held a pole to secure the hand position.

right arm and finger were shielded from view with an opaque piece of paper (not shown in the figure).

In one session, subjects took part in two experiments, each comprising 70 trials. During each trial, subjects were asked to sample a target force for a set time duration. They did so by pushing the index finger against a virtual spring, which determined the relationship between the positional displacement and force production. Each spring was simulated with the PHANTOM $^{\mathrm{TM}}$ by delivering a force tangential to the trajectory of the finger. Across trials, spring constants ranged from 50 to $100 \mathrm{~N} / \mathrm{m}$, and finger flexions at the 
target location ranged along an arc $30-40 \mathrm{~mm}$ in the Euclidean distance of the finger tip from 0 degrees. The variation in spring constants was intended to prevent subjects from being influenced by the terminal position of the finger or the range of forces experienced before the target force was reached.

As subjects pushed against the spring, a green feedback bar on the computer screen rose. The height of the green bar varied directly with the force produced by the subjects. Subjects were told to move the green bar into a target zone, represented by two red parallel lines intersecting the feedback bar. The target zone was designed so that the distance between the lines corresponded to $\pm 1.0 \%$ of the target force; thus the produced force was allowed to vary by this amount within the trial. For all 70 trials, the target force was 1.0, $1.5,2.0,2.5$, or 3.0 Newtons. In addition to the green feedback bar representing the produced force in a continuous format, the force level (levels 1 to 5 corresponding to the five different target forces) was spelled out and displayed on the screen.

Once the target zone was reached, the subject was asked to maintain the force for a designated time duration. The designated time duration was $2,4,6$, 8, or 10 seconds and it was also spelled out and displayed on the screen. At the end of that period, the feedback bar turned red, indicating that the subject could return the finger to the resting position at 0 degrees.

The subject then evaluated how much "effort" had been expended in maintaining the force in the target zone, using a scale from 1 to 10 . Effort was defined for the subject as the "sense of energy usage, exertion, or fatigue during the time that you were holding your finger against the haptic device." Special care was taken not to emphasize force or time in the experiment instructions. Subjects were given five example trials containing what were considered as requiring maximum effort (force level 5 for 10 seconds) and minimum effort (force level 1 for 2 seconds) before the set of experiments started. They were told that they should try to use the full scale of 1 to 10 given the range of trials experienced in the example trials.

In the first experiment of the session, subjects experienced a random sequence of 70 trials that sampled five discrete force and five discrete time levels. Because of the hardware limitation (overheating), the combination of force level 5 and time duration of 10 seconds was not included.

The second experiment was identical to the first except that the forces were distorted in some of the trials. The distortion levels were $0 \%, 7 \%, 15 \%$, and $22 \%$. For example, subjects had to produce 2.875 Newtons to get to the target zone on the feedback bar when the force level 4 (2.5 Newtons) was distorted by $15 \%$. Subjects were given the same visual feedback (both the bar and the spelled out force level and time duration) as if they produced 2.5 Newtons in undistorted trials. Distortion was randomly determined on a trial, with the constraint that all force/duration/distortion combinations were represented (except for force level 5 with a 22\% distortion and force level 5 with time duration of 8 and 10 seconds, again, due to the overheating problem). Subjects were not informed that the forces would be distorted, and no visual clues were given to indicate the existence of distortion. The time duration was never distorted in our experiment. By using the same subjects in both experiments, we gave them the opportunity to calibrate our feedback scale in an undistorted environment before we distorted the feedback.

In addition, five control trials were included at predesignated points in the experiment, during which subjects experienced force level 3 for 5 seconds. These trials were then used to gauge the subjects' fatigue levels, which may introduce a bias toward higher ratings as the experiment went on. Data from these trials were excluded from other analyses.

\subsection{Control experiment with no visual feedback}

Eight subjects ranged in age from 20 to 34 years participated in our control experiment. None of them had participated in the visual feedback experiments. The control experiment was 70 trials long and identical to the first experiment of the visual feedback session but without accurate visual feedback. The height of the target zone on the feedback bar was always at the same height regardless of the target force, and neither the force level nor the time duration was spelled out. Subjects were given five example trials containing what are considered as requiring maximum effort and minimum effort before the set of experiments started. Five control trials were included to gauge the fatigue levels that may cause biased ratings of effort.

\section{Results}

\subsection{Visual feedback experiment with no distortion}

Table 1 shows mean effort ratings by force and time. Because the experimental design was not fully factorial, multiple regression analysis was performed with mean effort (over subjects) as the dependent variable. We classified force and time levels as the independent variables. We found significant effects $(\mathrm{ps}<0.0001)$ of 
Table 1. Mean rated effort by force and time for the visual feedback experiment without feedback distortion.

\begin{tabular}{|c|c|c|c|c|c|}
\hline \multirow{2}{*}{$\begin{array}{c}\text { Time } \\
(\mathrm{sec})\end{array}$} & 1 & 1.5 & 2.0 & 2.5 & 3 \\
\cline { 2 - 6 } & 1.20 & 2.52 & 3.26 & 5.06 & 6.44 \\
\hline 2 & 1.89 & 2.48 & 4.11 & 5.26 & 7.56 \\
\hline 4 & 1.50 & 2.70 & 4.16 & 5.93 & 7.39 \\
\hline 6 & 1.70 & 3.00 & 4.67 & 5.61 & 7.92 \\
\hline 8 & 1.96 & 3.00 & 4.89 & 6.56 & \\
\hline 10 & \multicolumn{5}{|c|}{ Force (N) } \\
\hline
\end{tabular}

Table 2. Control trial results that validated the existence of fatigue effect for the visual feedback experiment without feedback distortion.

\begin{tabular}{|c|c|c|}
\hline $\begin{array}{c}\text { Trial } \\
\text { Number }\end{array}$ & $\begin{array}{c}\text { Mean } \\
\text { Rating }\end{array}$ & $\begin{array}{c}\text { Standard } \\
\text { Deviation }\end{array}$ \\
\hline 10 & 4.33 & 1.94 \\
\hline 22 & 4.11 & 1.36 \\
\hline 37 & 4.56 & 1.01 \\
\hline 49 & 4.11 & 1.36 \\
\hline 65 & 4.00 & 1.00 \\
\hline
\end{tabular}

both force and time in evaluating effort, while the effect of force was substantially greater. The standardized coefficient was 0.89 for force and 0.16 for time. The multiple variance accounted for $\left(\mathrm{R}^{2}\right)$ was 0.79 . To further quantify the effects, we performed simple linear regressions of effort against time at each level of force. All slopes and intercepts were greater than zero. Intercepts indicate the pure force effect and slopes indicate the pure time effect. ANOVAS showed intercepts were different significantly, $F(4,32)=33.30$, $p$ $<0.001$, but slopes did not differ significantly, $0.15>p$ $>0.10$.

The average ratings and their standard deviations of each of the control trials (i.e. force level 3 for 5 second duration) are shown in Table 2; they indicated no bias due to fatigue effects across the 70 trials.

\subsection{Visual feedback experiment with distortion}

Multiple regression analysis was again performed for the second experiment with the presence of distortion. We used mean effort as the dependent variable and force,

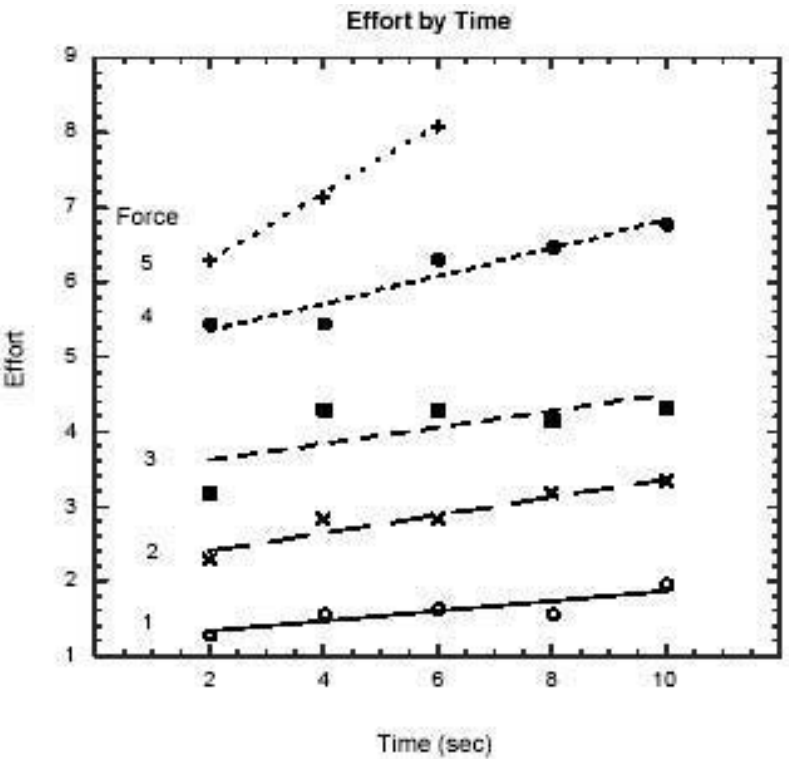

Figure 3. Simple linear regressions of effort against time at each force level in the visual feedback experiment with feedback distortion of force.

time, and distortion levels as the independent variables. There were significant effects of all three variables, $\mathrm{ps}<$ 0.0001 for force and time and $\mathrm{p}<0.05$ for distortion. As in the first experiment, the effect of force was the strongest contributor. The standardized coefficient was 0.99 for force, 0.18 for time, and 0.07 for distortion. The multiple $\mathrm{R}^{2}$ was 0.94 .

Simple linear regressions of effort against time at each level of force and corresponding regressions of effort against distortion at each level of force are shown in Figures 3 and 4 respectively. The mean slopes and intercepts of each force level are summarized in Table 3. The regressions of effort against time produced slopes that were significantly greater than 1.0 for all force levels and that increased with force, $F(4,32)=2.77$, p < 0.05 . The slopes of effort against distortion did not differ significantly overall, $\mathrm{p}>0.10$, and the $95 \%$ confidence intervals included zero for slopes of force levels 1,2, and 4. These results indicate that subjects weighted the visual feedback heavily over the actual force felt. The intercept effect in these simple regressions corresponded to the effect of force. The intercepts were significantly above zero in all 10 functions, and the intercept increased with force for both time and distortion, $\mathrm{F}(4,32)$ $=2.74$ and $4.36, \mathrm{p}<0.05$ and $<0.01$, respectively.

Control trial results for the visual feedback experiment with distortion were calculated and are summarized in Table 4: as in the case with no distortion, there is no evidence of fatigue-based rating changes over time. 


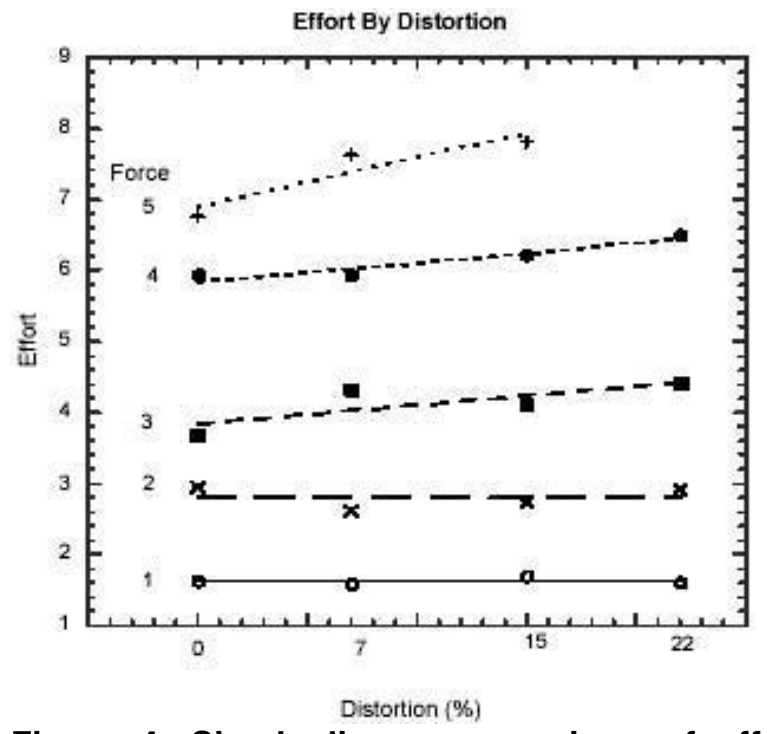

Figure 4. Simple linear regressions of effort against distortion at each force level in the visual feedback experiment with feedback distortion of force. One sample point contains all trials with the same force and distortion levels regardless of the time duration.

Table 3. Slopes and intercepts of simple regressions of effort against time and distortion, by force level in the visual feedback experiment with feedback distortion.

\begin{tabular}{|c|c|c|c|c|}
\hline \multirow{2}{*}{$\begin{array}{c}\text { Force } \\
(\mathrm{N})\end{array}$} & \multicolumn{2}{|c|}{ Time (sec) } & \multicolumn{2}{c|}{ Distortion (\%) } \\
\cline { 2 - 5 } & Slope & Intercept & Slope & Intercept \\
\hline 1.0 & 0.07 & 1.17 & 0.00 & 1.63 \\
\hline 1.5 & 0.12 & 2.14 & -0.00 & 2.85 \\
\hline 2.0 & 0.10 & 3.52 & 0.03 & 3.81 \\
\hline 2.5 & 0.19 & 4.98 & 0.03 & 5.86 \\
\hline 3.0 & 0.46 & 5.35 & 0.07 & 6.85 \\
\hline
\end{tabular}

Table 4. Control trial results that validated the existence of fatigue effect for the visual feedback experiment with feedback distortion.

\begin{tabular}{|c|c|c|}
\hline $\begin{array}{c}\text { Trial } \\
\text { Number }\end{array}$ & $\begin{array}{c}\text { Mean Effort } \\
\text { Rating }\end{array}$ & $\begin{array}{c}\text { Standard } \\
\text { Deviation }\end{array}$ \\
\hline 6 & 4.78 & 1.09 \\
\hline 18 & 4.56 & 1.13 \\
\hline 26 & 4.11 & 1.17 \\
\hline 46 & 3.78 & 1.39 \\
\hline 67 & 4.00 & 1.80 \\
\hline
\end{tabular}

Table 5. Mean rated effort by force and time for the control experiment with no visual feedback.

\begin{tabular}{|c|c|c|c|c|c|}
\hline \multirow{2}{*}{$\begin{array}{c}\text { Time } \\
(\mathrm{sec})\end{array}$} & 1 & 1.5 & 2.0 & 2.5 & 3 \\
\hline 2 & 2.45 & 3.50 & 3.83 & 6.06 & 6.88 \\
\hline 4 & 3.38 & 2.54 & 6.13 & 5.17 & 7.88 \\
\hline 6 & 2.31 & 3.42 & 4.70 & 6.58 & 5.94 \\
\hline 8 & 2.46 & 4.06 & 5.75 & 5.44 & 7.25 \\
\hline 10 & 3.54 & 4.25 & 5.75 & 7.13 & \\
\hline
\end{tabular}

\subsection{Control experiment with no visual feedback}

Mean effort for the control experiment is shown in Table 5 by force and time. Multiple regression, with mean effort as the dependent variable and force and time levels as the independent variables, showed significant effects of both, $\mathrm{p}<0.0001$ for force and $\mathrm{p}<0.05$ for time. The standardized coefficient was 0.92 for force and 0.10 for time, and the multiple $R^{2}$ was 0.85 . These results are comparable to the results found during the experiment with visual feedback. Simple linear regressions of effort against time at each level of force showed that all intercepts are above zero and $\mathrm{F}$ test is highly significant, $\mathrm{F}(4,28)=31.20, \mathrm{p}<0.001$. The slopes for force levels 1 to 5 were $0.06,0.15,0.17,0.12$, and -0.04 respectively, making only the slope of force level 3 significant.

\section{Discussion}

The results of all experiments showed that the feedback about the force level was by far the most powerful predictor of perceived physical effort when people exerted force against a virtual spring. Distortions that increased the force production by as much as $22 \%$ had little influence. The influence of stated force level can be seen clearly by comparing the force distorted by $22 \%$ at one level to those that are undistorted at the next level in Figure 4. For example, force level 3 with $22 \%$ distortion and force level 4 with $0 \%$ distortion are discretely different in perceived effort level while the forces produced are very similar (2.44 and 2.5 Newtons). In fact, the forces felt are ordered in reverse of the levels for force level 4 with $22 \%$ distortion (3.05 Newtons) and force level 5 with no distortion (3.0 Newtons) yet the stated force level dominated the perceived effort.

It is surprising that although subjects had equivalent feedback about force and time, the effect of force was 
much stronger. Results from the control experiment without the visual feedback reconfirm that subjects tend to weigh force more than time when determining physical effort. The time intervals used here should have been distinguishable on a relative if not absolute basis [5]. We predict, however, that if the time durations were much longer, subjects would make a distinction in the perceived effort level. This is a useful result that can be transferred to the rehabilitation paradigm.

For the experiments with visual feedback, the effect of time (the slopes of the linear regression curves) was more prominent as the target force level increased. This effect was not present for the experiment without visual feedback. Given that people are very good at telling the time without any feedback [5], the difference between these experiments indicate that subjects may have simply used the time duration indicated on the monitor toward the effort rating even though they did not truly feel much difference on their finger.

The effect of distortion is best expressed in the slopes of linearly regressed curves in Figure 4. While the slopes were not significantly different from each other and three out of five curves have slopes close to zero, the curves generally appear as if the distortion effect is larger for larger force levels. This appearance of larger distortion effect, however, is based on absolute differences in force rather than percent distortion. The slope of these fitted lines represent, at most, less than one tenth of one Newton's difference between distortion levels. It would be interesting to investigate the distortion effect of absolute force rather than percent force.

We find it encouraging that there was no evidence of rating biases based on fatigue in the experiments. Over the one-hour session, subjects' ratings of control trials remained consistent, to the extent that they were able to rate trials with identical force/time information consistently. Consistency was also high across experiments (compare Tables 2 and 4), not only with respect to the mean but also with respect to variability. This constancy of performance level suggests that in a rehabilitation context, we may be able to use distorted feedback for an extended period of time without the interference by cognitive level factors such as perceived physical effort.

People's sensitivity to force varies significantly [4] and a weaker subject might become fatigued earlier than a stronger subject. This statement would be even stronger in applications of feedback distortion for the disabled. In the case where the users' abilities differ greatly, the amount of force experienced will have to be scaled to patient ability. The data from these experiments show that all subjects were affected by the visual distortion, and thus we expect that methods similar to those described above would be transferable to disabled subjects.

By having the same subjects participate in experiments with and without feedback distortion, we intended that they calibrate the feedback during no distortion condition experienced first. However, it could be argued that the consistent feedback in the first experiment led them to ignore the distortions in the second. Two considerations that argue against this are that in other work, we have confirmed subjects' strong reliance on distorted feedback [9], and that our results presented here show that consistent feedback about time had only a modest influence on subjects' effort ratings. Thus the statement of a variable's value does not guarantee an effect of that variable.

In this paper, we showed that higher-level factors such as perceived physical effort are affected by feedback distortion. Within the bound of extended JNDs under visual feedback distortion [9], the perceived effort is strongly linked to the visual feedback of force rather than the actual force produced by the subjects. This result is encouraging for creating a virtual environment for rehabilitation that uses feedback distortion as a way to train patients to produce more force and move further without their awareness.

\section{References}

[1] S. Allin, Y. Matsuoka, and R. Klatzky, "Measuring Just Noticeable Differences For Haptic Force Feedback: A Tool for Rehabilitation", Proceedings of the 10th IEEE Symposium on Haptic Interfaces for Virtual and Teleoperator Systems (HAPTICS 2002), 2002, pp. 299 - 302.

[2] G. Borg, Borg's perceived exertion and pain scales, viii, 104, Human Kinetics, Champaign, IL. 1998.

[3] W. Cain, "Nature of perceived effort and fatigue: Roles of strength and blood flow in muscle contractions", Journal of Motor Behavior, 1973, 5, pp. 33 - 47.

[4] F. J. Clark and K. W. Horch, "Kinesthesia", In K.R. Boff, L. Kaufman, \& J. P. Thomas (Eds.), Handbook of Perception \& Human Performance, Vol. 1 Sensory Processes and Perception (pp. 13-1-13-62), John Wiley \& Sons. New York. 1986.

[5] H. Eisler, "Experiments on subjective duration 1868-1975: A collection of power function exponents", Psychological Bulletin, 1976, 83, pp. 1154 - 1171.

[6] E.A. Fleishman, D.L. Gebhardt, and J.C. Hogan, "The Measurement of Effort", Ergonomics, 1984, 27, pp. 947 - 954. 
[7] J.C. Hogan, G.D. Ogden, D.L. Gebhardt, and E.A. Fleishman, "Reliability and Validity of Methods for Evaluating Perceived Physical Effort", Journal of Applied Psychology, 1980, 65, pp. 672 - 679.

[8] F. Lu, "Perceived exertion: Integration of physiological and psychological factors", Dissertation Abstracts International Section A: Humanities \& Social Sciences, 1995, 56(2-A).

[9] Y. Matsuoka, S. Allin, and R. Klatzky, "The Tolerance for Visual Feedback Distortions in a Virtual Environment", Physiology and Behavior, 2002, 77(4-5) pp.651 - 655.

[10] J. G. Williams, R.S. Purewal, "Development and initial validation of the Effort Sense Rating Scale (ESRS): A selfperceived index of physical fitness.", Preventive Medicine: An International Journal Devoted to Practice \& Theory, 2001, 32(2), pp.103 - 108. 